\title{
Sequence Analysis of the K13-Propeller Gene in Artemisinin Challenging Plasmodium falciparum Isolates from Malaria Endemic Areas of Odisha, India: A Molecular Surveillance Study
}

\author{
Ramakanta Rana $\mathbb{D}^{1},{ }^{1}$ Manoranjan Ranjit $\mathbb{D}^{1},{ }^{1}$ Madhusmita Bal, $^{1}$ Hemant Kumar Khuntia, ${ }^{1}$ \\ Sanghamitra Pati $\mathbb{C}^{1},{ }^{1}$ Sri Krishna, ${ }^{2}$ and Aparup Das $\mathbb{1}^{2}$ \\ ${ }^{1}$ Division of Molecular Epidemiology and Public Health, ICMR-Regional Medical Research Centre, Bhubaneswar, Odisha, India \\ ${ }^{2}$ Division of Vector Borne Diseases, ICMR-National Institute of Research in Tribal Health, Jabalpur, India \\ Correspondence should be addressed to Aparup Das; aparupdas@nirth.res.in
}

Received 22 July 2019; Accepted 12 February 2020; Published 16 March 2020

Academic Editor: Rana Chattopadhyay

Copyright (c) 2020 Ramakanta Rana et al. This is an open access article distributed under the Creative Commons Attribution License, which permits unrestricted use, distribution, and reproduction in any medium, provided the original work is properly cited.

\begin{abstract}
Estimation of the spread and advancement of Plasmodium falciparum artemisinin-resistant parasites can be done by probing polymorphisms in the kelch (Pfk13) domain (a validated molecular marker). This study aimed to provide baseline information for future artemisinin surveillance by analyzing the k13-propeller domain in $P$. falciparum field isolates collected from 24 study areas in 14 malaria hot spots of Odisha (previously Orissa) during July 2018-January 2019. A total of 178 P. falciparum mono infections were assessed. An 849-base pair fragment encoding the Pfk13 propeller was amplified by nested polymerase chain reaction and sequenced in both directions (PCR). After DNA alignment with the 3D7 reference sequence, all samples were found to be wild type. It can be anticipated that malaria public health is not under direct threat in Odisha relating to ART resistance.
\end{abstract}

\section{Introduction}

Malaria infection by Plasmodium falciparum is creating a major public health burden all over the world particularly in tropical and subtropical areas. After the clinical failure of Chloroquine and Sulphadoxine-Pyrimethamine due to the occurrence of resistance in South East Asian region and many more countries, Artemisinin or Artemisinin Combination Therapy (ACT) has been recommended by the World Health Organizations (WHO) as the first-line treatment for uncomplicated P. falciparum malaria since 2006 [1]. Further continuous reuse of this single ACT resulting in P. falciparum-resistant alleles to Artemisinin or its derivatives has been reported for the first time in Western Cambodia and Thailand border [2, 3]; more interestingly, these resistant isolates might be carried by the parasites distributed rapidly across all SE Asian countries and also some parts of Africa [4, 5]. Both Artemisinin Combination Therapies (ACTs) and its derivatives along with partner drug resis- tance to $P$. falciparum isolates have threatened the current efforts for the reduction of the burden of infectious malaria all over the world $[6,7]$

As per the WHO guidelines, the definition of the emerged resistant alleles across Thai and Cambodia border has been characterized by $[8,9]$ not only with reduced parasite clearance rate and increasing parasite clearance halflife or survived young ring stage parasites but also with the continuation of parasites on the third day of ACTs $[1,10-$ 18]. These worldwide problems give rise to several approaches for the intense surveillance and detection of Artemisinin-resistant falciparum parasites including molecular marker evaluation [7, 19]. Genome-wide association studies of the $P$. falciparum whole genome sequencing data have identified a gene named PfKelch13-PF3D7-1343700 locating on chromosome 13 which encodes K13-propeller protein. The clinical ART failure has been emerged independently by the occurrence of nonsynonymous mutations or polymorphisms in the beta-propeller domain of that kelch 
protein mutation-expressed substituted AA sequence. But still the question remains unreciprocated why the alleles get transformed into a resistant form and whether they spread geographically to other adjacent countries. In Southeast Asia, Ariey and coworkers [20] have detected some alleles of the K13 propeller genes (C580Y, Y493H, R539T, and I543T) to display delayed parasite clearance. And the rest of the 9 candidate resistance mutations, i.e., $\mathrm{P} 441 \mathrm{~L}$, F446I, G449A, N458Y, P553L, R561H, V568G, P574L, and $\mathrm{A} 675 \mathrm{~V}$, also were detected by several group of researchers $[4,16,20-23]$.

On one hand, in the year 2010, the Government of India launched the National Malaria Control Policy for the complete eradication of malaria infection starting from the rural level by providing LLINs and ACT kits to the village-level health workers ASHA; and the other hand, recent detection of the low frequency, candidate gene mutations of Kelch13 G625R, R539T in certain regions of North Eastern India has put in danger the eradication program. The recent researchers from India could not highlight completely whether these mutant alleles are able to repress the protein expression or causing direct clinical Artemisinin failure $[9,24,25]$ remains a research question to be evaluated through molecular genotyping of K13 polymorphism.

The percentage of Plasmodium falciparum malaria has been drastically reduced in the year 2018 (81\%) as compared with 2012 (93\%) in Odisha, which previously remained one of the malaria-endemic state contributing to $40 \%$ (until 2017) of the total malaria burden of India [26]. The state geographically lies adjacent to the North-Eastern regions of India. The presence of the above low frequency and nonsynonymous candidate mutation of k13-resistant alleles, whether involved in clinical ART failure in this state or not, could not be ignored in the routine surveillance of falciparum malaria for the complete malaria elimination approach in Odisha, India.

\section{Materials and Methods}

2.1. Ethical Approval. All the methods of this molecular surveillance study was reviewed and approved by the Institutional Human Ethics Committee of ICMR-RMRC Bhubaneswar, Odisha, India, in accordance with relevant guidelines and regulations of Indian Council of Medical Research (ICMR). The informed consent was obtained from all individual patients prior to blood collection.

2.2. Study Area, Sample, and Demographic Data Collection. The study was carried out in both urban and remote areas of the different districts of Odisha, India. 16 sampling sites were selected from localities which were surrounded by dense forest and inaccessible areas, and 9 areas from urban areas as shown in Table 1. The present study was carried out from July 2018 to January 2019. Finger prick bloods of both asymptomatic and symptomatic patients were evaluated for Plasmodium falciparum malaria parasites by field-based rapid immunochromatographic test. Patients having $\mathrm{Pf} / \mathrm{Pv}$-positive cases were included in this study, and RDT-negative cases were excluded from this study. After obtaining the written informed consent, $1 \mathrm{ml}$ of intravenous blood was collected from the patients ICT positive for $\mathrm{Pf} / \mathrm{Pv}$ and stored in EDTA vial.

2.3. Parasitic DNA Extraction and Genotyping. Parasitic DNA was isolated by using proteinase $\mathrm{K}$ and phenol method with slight modifications as described by Sambrook et al.'s molecular cloning and stored at $-20^{\circ} \mathrm{C}$ until use [27].

2.4. Nested PCR Amplification. Species-specific nested PCR amplification was carried out to screen the Plasmodium falciparum malaria parasites using 18srRNA genes as described in $[28,29]$. The PfKelch13 gene fragment was amplified by nested PCR protocols reported previously by F. Ariey et al. with modifications [20]. The quality and concentration of all the PCR products were analyzed by using 1.5\% agarose gel electrophoresis following ethidium bromide stains. The details of nested PCR primers and conditions for the PfKelch 13 conditions are shown in Table 2.

2.5. DNA Sequencing. Successful PCR products showing single band in gel were then subjected to PCR purification (using Fast ${ }^{\mathrm{AP}}$ alkaline phosphatise and exonuclease I) and further processed for DNA sequencing by Sanger methods (an in-house facility of ICMR-NIRTH, Jabalpur) with $2 \mathrm{x}$ coverage (sequenced from both the forward and reverse directions). Briefly, the amplified fragments were sequenced using Big Dye Terminator cycle sequencing ready reaction version 3.1 and ABI Prism DNA sequence Analyzer3130 $[17,20]$. For each isolate, sequence chromatograms were viewed carefully, and all the sequences from a single population were aligned (with the help of Gene Doc multiple Sequence Alignment Editor and shading utility version 2.7.000) alongside the reference Pfkelch13 sequence.

\section{Results}

During the study period, a total of 178 patients (aged 4 to 78 years) were enrolled and diagnosed with Plasmodium falciparum by RDT kit and molecular tests shown in Tables 1 and 3. 175 (98.31\%) positive cases were detected by RDT and 128 (73.14\%) were confirmed Plasmodium falciparum through nested PCR diagnosis, by using published primers (rPLU6, rPLU5 primary PCR, and rFAL1 and rFAL2 as nested primer for falciparum infections) [29]. 72\% (93/128) of the K13-propeller gene was amplified by nested PCR method as described in [20] and the thermo cycling conditions shown in Table 2. 57 PfK13-propeller genes were successfully sequenced in the selected 64 samples. Samples that could not be amplified were excluded from sequencing; by using this approach, out of 64 monoinfected $P$. falciparum isolates, only $57(89.96 \%)$ could be successfully sequenced for the 849 nucleotide base pair DNA fragment of the Pfk13 gene. After sequence alignment with the 3D7 reference sequence, all samples were found to be wild type. The nonavailability of novel K13 mutations (neither validated nor candidate) associated with ART resistance to malaria parasites suggested no risk for the Artemisinin Combination Therapy (ACT), in Odisha. 


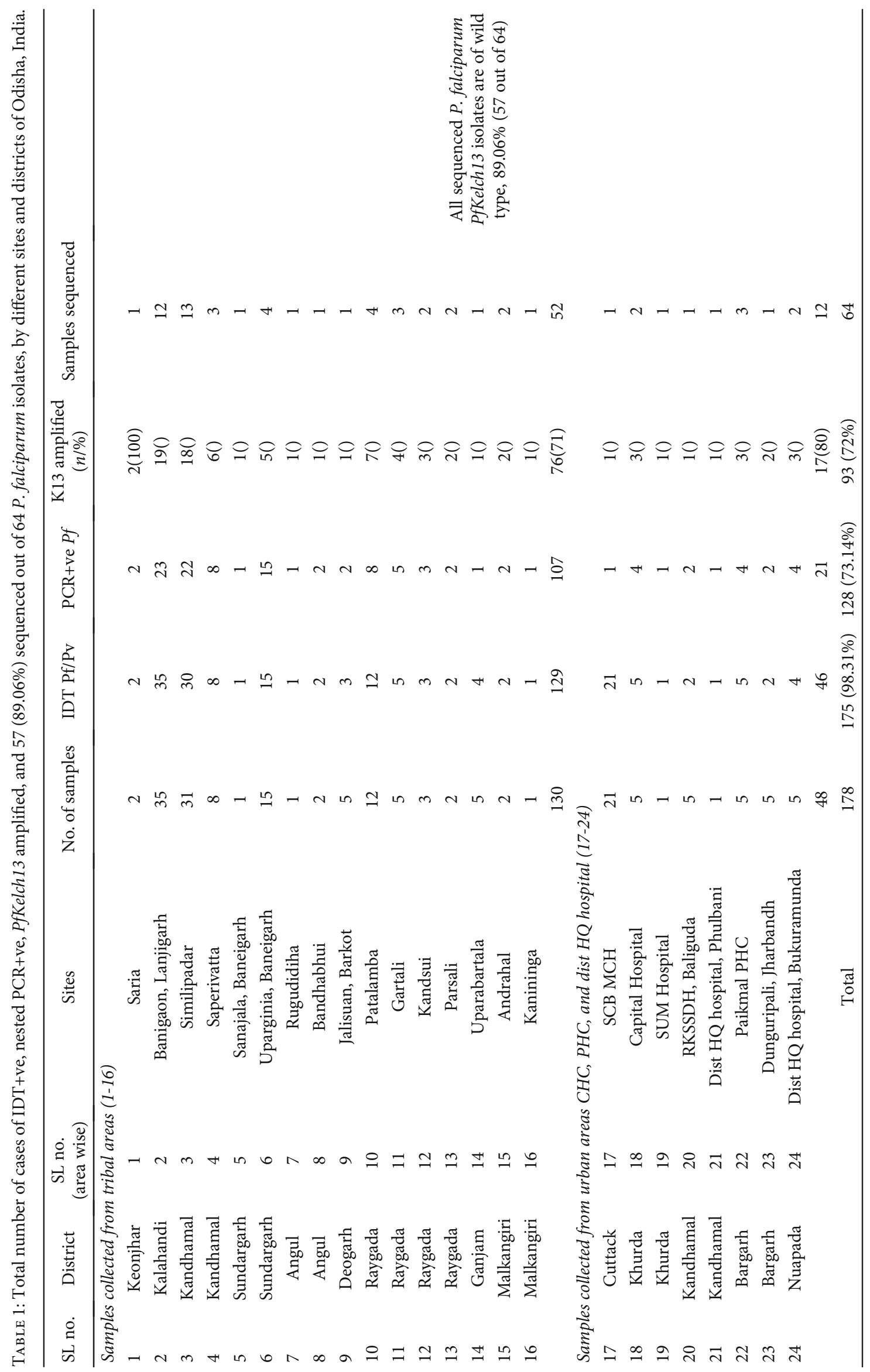




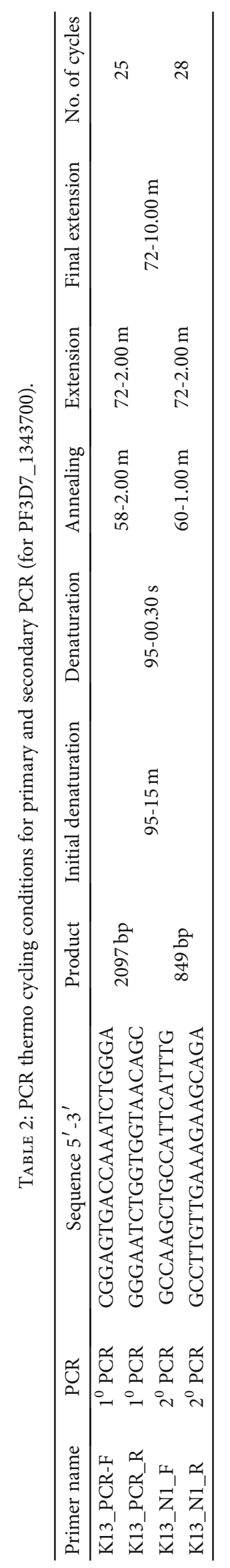


TABLE 3: Distribution of $P$. falciparum isolates age wise, gender wise, and k13 sequencing results.

\begin{tabular}{lccccccc}
\hline Age group & $\begin{array}{c}\text { Frequency of } \\
\text { Pf cases }\end{array}$ & $\%$ & $\begin{array}{c}\text { Pf cases in } \\
\text { male }\end{array}$ & K13 male & $\begin{array}{c}\text { Pf cases in } \\
\text { female }\end{array}$ & K13 female & \\
\hline $0-9$ & 36 & 28.12 & 27 & 20 & 9 & 5 & \\
$10-19$ & 26 & 20.31 & 12 & 8 & 14 & 5 & \\
$20-29$ & 13 & 10.15 & 5 & 5 & 8 & 6 & \\
$30-39$ & 9 & 7.03 & 4 & 4 & 5 & 4 & K13 selected for Propeller sequenced/results \\
$40-49$ & 24 & 18.75 & 17 & 12 & 7 & 5 & sequencing no. 64 (no-wild type, 57 wild type) \\
$50-59$ & 14 & 10.93 & 7 & 7 & 7 & 6 & \\
$60-69$ & 5 & 3.9 & 2 & 2 & 3 & 3 & \\
$70-79$ & 1 & 0.78 & 0 & 0 & 1 & 1 & \\
Total & 128 & & $74(57.8 \%)$ & 58 & $54(42.1 \%)$ & 35 & \\
\hline
\end{tabular}

\section{Discussions and Conclusion}

Currently, ACT is the only WHO-recommended antimalarial drug to combat the severity of Plasmodium falciparum infection across the globe including Odisha, India. Not only is the emergence and spread of ART-resistant isolate in South East Asian regions a matter of concern but also the occurrence of low-frequency candidate mutations in the k13-propeller gene across certain regions of North-Eastern India could draw the state of Odisha's attention. Since there is no report of transformation of sensitive to resistant alleles reported, molecular evolutionary patter is not known yet in this area of Odisha.

Prior articles from India could not put emphasis on the geographical distribution of validated k13 mutant alleles [9], but the above presented mutations probably create the ACT regimen in high risk for the uncomplicated falciparum malaria in the study areas of Odisha. As Odisha is aiming to reduce malaria particularly Plasmodium falciparum infections completely in 2020 to become a malaria-free state, RDT kits and ACTs have been provided to the village-level ASHA workers under the joint action of National Health Mission-National Vector Borne Diseases (NHM-NVBDCP) $[26,27]$. Unfortunately, there is no alternative to the current regimen ACT for the severe uncomplicated malaria and also no significant research has yet been done on K13-propeller polymorphism; hence, it is essential to monitor the efficacy of the Artemisinin derivative through molecular surveillance study in the selected areas of the state during the year 2018. Recent study aims to track the research questions raised for the surveillance of falciparum infections by the PCR-based sequencing analysis of Pfkelch13 gene propeller polymorphism.

None of the mutations in the $\mathrm{k} 13$ gene whether validated or candidate marker has originated from the current study. Furthermore, the nonoccurrence of resistant alleles in Odisha defines against the contention that the resistant alleles might be distributed in other geographically closer state(s) to Odisha. To the best of our knowledge, this is the first report of a study in Odisha, India, that investigates the efficacy of usual ART resistance through the detection of the Pfkelch13 gene propeller polymorphism study.

Though it may be interpreted from this study that no direct threat to the consequence of ART on the falciparum isolates in Odisha, more attention is required on account of the surveillance study for the complete elimination of malaria in Odisha. Field-based survey along with low-cost molecular diagnosis, genotyping of markers related to causing clinical failure of ART should be carried out along with a large number of sample sizes.

\section{Data Availability}

The data used to support the findings of this study are available from the corresponding author upon request.

\section{Conflicts of Interest}

The authors declare that they have no conflicts of interest regarding the publication of this manuscript.

\section{Acknowledgments}

RKR thanks the Indian Council of Medical Research, New Delhi, for awarding Senior Research Fellowship (ICMR-SRF). RKR and MR thank the director of ICMRRMRC, Bhubaneswar, for the constant encouragement and providing the necessary laboratory facilities during the first phase of the work. We are grateful to all patients and their families, who participated in this study. We thank Dr. Praveen Kumar Bharti (Scientist-E) ICMR-NIRTH for providing the necessary laboratory facilities during the sequencing work.

\section{References}

[1] World Health Organization, Guidelines for the Treatment of Malaria. 3rd edition, World Health Organization, Geneva, 2015.

[2] J. P. Daily, "K13-propeller mutations and malaria resistance," The New England Journal of Medicine, vol. 374, no. 25, pp. 2492-2493, 2016.

[3] A. M. Dondorp, F. Nosten, P. Yi et al., "Artemisinin resistance inPlasmodium falciparumMalaria," New England Journal of Medicine, vol. 361, no. 5, pp. 455-467, 2009.

[4] M. Ocan, D. Akena, S. Nsobya et al., "K13-propeller gene polymorphisms in Plasmodium falciparum parasite population in malaria affected countries: a systematic review of prevalence 
and risk factors," Malaria Journal, vol. 18, no. 1, pp. 1-17, 2019.

[5] K. Haldar, S. Bhattacharjee, and I. Safeukui, "Drug resistance in _Plasmodium_," Nature Reviews Microbiology, vol. 16, no. 3, pp. 156-170, 2018.

[6] O. Miotto, J. Almagro-Garcia, M. Manske et al., "Multiple populations of artemisinin-resistant Plasmodium falciparum in Cambodia," Nature genetics, vol. 45, no. 6, pp. 648-655, 2013.

[7] N. Thuy-Nhien, N. K. Tuyen, N. T. Tong et al., "K13 propeller mutations in Plasmodium falciparum populations in regions of malaria endemicity in Vietnam from 2009 to 2016," Antimicrobial Agents and Chemotherapy, vol. 61, no. 4, pp. 1-10, 2017.

[8] World Health Organization, Global Malaria Programme, status report on artemisinin resistance, and artemisinin based combination therapy efficacy, WHO, 2018, August, 2018, https://www.who.int/malaria/publications/atoz/artemisininresistance-august2018/en/.

[9] S. Das, S. Manna, B. Saha, A. K. Hati, and S. Roy, "Novel pfkelch13 gene polymorphism associates with artemisinin resistance in Eastern India," Clinical Infectious Diseases, vol. 69, pp. 144-1152, 2019.

[10] B. Hanboonkunupakarn and N. J. White, "The threat of antimalarial drug resistance," Tropical Diseases Travel Medicine and Vaccines, vol. 2, no. 1, pp. 1-5, 2016.

[11] V. Duru, B. Witkowski, and D. Ménard, "Plasmodium falciparum resistance to artemisinin derivatives and piperaquine: a major challenge for malaria elimination in Cambodia," The American Journal of Tropical Medicine and Hygiene, vol. 95, no. 6, pp. 1228-1238, 2016.

[12] Q. Cheng, D. E. Kyle, and M. L. Gatton, "Artemisinin resistance in Plasmodium falciparum: a process linked to dormancy?," International Journal for Parasitology: Drugs and Drug Resistance, vol. 2, no. 2012, pp. 249-255, 2012.

[13] N. J. White, "Antimalarial drug resistance," The Journal of Clinical Investigation, vol. 113, no. 8, pp. 1084-1092, 2004.

[14] A. M. Thu, A. P. Phyo, J. Landier, D. M. Parker, and F. H. Nosten, "Combating multidrug-resistant Plasmodium falciparum malaria," The FEBS Journal, vol. 284, no. 16, pp. 2569-2578, 2017.

[15] A. P. Phyo, S. Nkhoma, K. Stepniewska et al., "Emergence of artemisinin-resistant malaria on the western border of Thailand: a longitudinal study," The Lancet, vol. 379, no. 9830, pp. 1960-1966, 2012.

[16] C. J. Woodrow and N. J. White, "The clinical impact of artemisinin resistance in Southeast Asia and the potential for future spread," FEMS Microbiology Reviews, vol. 41, no. 1, pp. 34-48, 2017.

[17] M. A. Oboh, D. Ndiaye, H. A. Antony et al., "Status of Artemisinin Resistance in Malaria Parasite Plasmodium falciparum from Molecular Analyses of the Kelch13 Gene in Southwestern Nigeria," Bio Med Research International, vol. 2018, article 2305062, pp. 1-5, 2018.

[18] B. Witkowski, C. Amaratunga, N. Khim et al., "Novel phenotypic assays for the detection of artemisinin-resistant Plasmodium falciparum malaria in Cambodia: in-vitro and ex-vivo drug-response studies," The Lancet Infectious Diseases, vol. 13, no. 12, pp. 1043-1049, 2013.

[19] O. Miotto, J. Almagro-Garcia, M. Manske et al., "Multiple populations of artemisinin-resistant Plasmodium falciparum in Cambodia," Nature Genetics, vol. 45, no. 6, pp. 648-655, 2013.

[20] F. Ariey, B. Witkowski, C. Amaratunga et al., "A molecular marker of artemisinin-resistant Plasmodium falciparum malaria," Nature, vol. 505, no. 7481, pp. 50-55, 2014.

[21] B. Blasco, D. Leroy, and D. A. Fidock, "Antimalarial drug resistance: linking Plasmodium falciparum parasite biology to the clinic," Nature Medicine, vol. 23, no. 8, pp. 917-928, 2017.

[22] L. R. Gomes, A. Lavigne, C. L. Peterka et al., "Absence of K13 polymorphism in Plasmodium falciparum from Brazilian areas where the parasite is endemic," Antimicrobial Agents and Chemotherapy, vol. 62, no. 10, pp. 1-4, 2018.

[23] Y. He, S. Campino, E. D. Benavente et al., "Artemisinin resistance-associated markers in Plasmodium falciparum parasites from the China-Myanmar border: predicted structural stability of $\mathrm{K} 13$ propeller variants detected in a low-prevalence area," PLoS One, vol. 14, no. 3, pp. e0213613-e0213686, 2019.

[24] S. Das, B. Saha, A. K. Hati, and S. Roy, "Evidence of artemisinin-resistant Plasmodium falciparum malaria in Eastern India," The New England Journal of Medicine, vol. 379, no. 20, pp. 1962-1964, 2018.

[25] N. Mishra, R. S. Bharti, P. Mallick et al., "Emerging polymorphisms in falciparum Kelch13 gene in North- eastern region of India," Malaria Journal, vol. 15, no. 1, pp. 1-6, 2016.

[26] G. Dhangadamajhi, R. K. Hazra, and M. R. Ranjit, "Malaria in Odisha and future perspectives," The Journal of Infectious Diseases, vol. 114, pp. 289-304, 2015.

[27] J. S. Brook, D. W. Russell, and M. R. Green, Molecular Cloning-A Laboratory Manual, vol. 1, Cold spring Harbor Laboratory Press, New York, 3rd edition, 2001.

[28] G. Snounou, S. Viriyakosol, W. Jarra, S. Thaithong, and K. N. Brown, "Identification of the four human malaria parasite species in field samples by the polymerase chain reaction and detection of a high prevalence of mixed infection," Molecular and Biochemical Parasitology, vol. 58, no. 2, pp. 283-292, 1993.

[29] G. Snounou, S. Viriyakosol, Xin Ping Zhu et al., "High sensitivity of detection of human malaria parasites by the use of nested polymerase chain reaction," Molecular And Biochemical Parasitology, vol. 61, no. 2, pp. 315-320. 